Voix et Images

voixetimages

\title{
Apprendre de la disparition
}

\section{Denise Brassard}

Volume 44, numéro 3 (132), printemps-été 2019

URI : https://id.erudit.org/iderudit/1064631ar

DOI : https://doi.org/10.7202/1064631ar

Aller au sommaire du numéro

Éditeur(s)

Université du Québec à Montréal

ISSN

0318-9201 (imprimé)

1705-933X (numérique)

Découvrir la revue

Citer ce compte rendu

Brassard, D. (2019). Compte rendu de [Apprendre de la disparition]. Voix et Images, 44(3), 201-210. https://doi.org/10.7202/1064631ar d'utilisation que vous pouvez consulter en ligne.

https://apropos.erudit.org/fr/usagers/politique-dutilisation/ 


\author{
P O É S I E \\ Apprendre de la disparition \\ $+++$
}

DENISE BRASSARD

Université du Québec à Montréal

Si l'on en juge par la réception critique dont elles jouissent depuis quelque temps, il semble que les poètes innues aient le vent dans les voiles. Et pour cause. Je vous ai parlé de Joséphine Bacon dans ma dernière chronique, je vous parlerai maintenant de Marie-Andrée Gill ${ }^{1}$. Comme il avait déjà fait pas mal parler de lui et que je me méfie de l'effet de mode, j'avais d'abord mis ce recueil de côté. Mais il revenait invariablement au-dessus de la pile... Alors soit!

Depuis que j'ai découvert Marie-Andrée Gill, je me demande comment une poésie aussi désarmante de simplicité peut être aussi percutante. Est-ce un simple effet de réminiscence? Si cet ancrage dans ma culture et mes paysages d'origine détermine certes ma lecture, il ne suffit pas à expliquer le succès de l'auteure, dont le lectorat déborde largement le Saguenay-Lac-Saint-Jean. Mais c'est peut-être au fond cet amour du simple et l'art de le conjuguer qui touchent autant les lecteurs. En cela la jeune poète pourrait figurer parmi les Gérald Godin et Hélène Monette de nos bibliothèques.

Chauffer le dehors nous plonge au cœur d'un deuil amoureux, celui d'un amour impossible et néanmoins dévorant:

C'est une histoire d'amour comme toutes mes autres

un autobus écrit spécial

avec personne dedans. (19)

Puisqu'il n'y a rien d'autre à faire que d'attendre que ça passe, que la blessure guérisse, rien à faire en somme que du mort avec du vivant, en espérant que les nouvelles pousses se nourriront des cadavres, aussi bien essayer de comprendre d'où vient et où va cet autobus. La réflexion sur le sens de cette déchirure - qu'est-ce qui fait qu'un être vous est cher au point que sa proximité vous donne le vertige et que son absence crée un vide abyssal? - est aussi un questionnement sur ce qui nous donne le sentiment d'être vivant, dont l'écriture.

1 Laquelle d'ailleurs cite son aînée en épigraphe de son dernier livre: «J'ignore si demain me gardera intacte/ Je dis que l'espoir de se laisser être/Éloigne le désespoir - Joséphine Bacon», Marie-Andrée Gill, Chauffer le dehors, Chicoutimi, La Peuplade, coll. «Poésie», 2019, p. 5. 
La sensibilité, tendue à l'extrême, s'exprime à travers une attention aiguë aux gestes du quotidien, aux objets banals, aux rituels de rien. C'est une manière d'accueillir, sans discrimination, ce qui porte le sens, en même temps qu'une façon de dédramatiser, de faire acte d'humilité, bref, de ne pas se complaire dans la blessure narcissique. Conséquemment, une constante circulation entre intérieur et extérieur structure le recueil. En plus de favoriser la respiration et de garder le sujet en contact avec le vivant, cette circulation donne lieu à des revirements sémantiques. «Chauffer le dehors» est une expression généralement connotée négativement: on l'emprunte pour désigner un gaspillage d'énergie. Or il est des gaspillages nécessaires, des impératifs somptuaires dont on ne saurait faire l'économie. Il en va de l'amour comme de la poésie:

\author{
L'amour c'est une forêt vierge \\ pis une coupe à blanc \\ dans la même phrase. (11)
}

Nombreuses sont les expressions populaires, généralement dévalorisées ou à connotation négative, dont la teneur figurative est exploitée et le sens ainsi tourné au positif. La circulation entraîne également le mélange des cultures, des espaces et des âges de la vie:

\author{
T'es la talle d'épinettes noires \\ qui brûle mon cœur full de super \\ C'est juste impossible que tu viennes plus \\ t'abreuver à mon esprit ancestral \\ de crème soda. (12)
}

Si l'on devait associer un âge à la poésie de Marie-Andrée Gill, ce serait probablement l'adolescence, cet âge mal ajointé aux temps et cependant relié à tous. Véritable anachronisme, l'adolescence nous permet de remonter à la nuit des temps tout en nous projetant dans le futur. Constamment tendue par le désir, impatiente, l'adolescente doit néanmoins ralentir la marche, sentir le territoire, y faire ses marques, se positionner, si elle veut mettre au jour sa propre pensée. Or c'est précisément ce que fait Gill dans sa poésie: opposer la lenteur à la vitesse et la patience à la fulgurance, non pour permettre à l'une de dominer l'autre, mais pour les faire cohabiter. Le cœur pointé vers le nord, elle tente le possible:

Quelque chose en moi garde la lampe allumée une déchirure, pas tout à fait une blessure, plutôt comme quand les nuages s'ouvrent là au milieu, entre les poumons - une envie qui peut pas s'empêcher de chercher le trouble, provoquer la rencontre, essayer n'importe quoi tout à coup que. (17) 
Le livre fait une place importante à la culture populaire. Les "toune[s] de Céline» (62) y côtoient les références littéraires sans pâlir, ces dernières étant traitées de telle sorte qu'aucune hiérarchie ne s'établit entre elles². D'ailleurs, aucune hiérarchie ne tient dans cet univers où tout est relié et vibre au même diapason: la montagne qui prend «sa marche sur les millénaires» (82), le chat qui entre et sort de la maison, les meubles qu'elle change de place, «les brûlots qui éclosent encore dans [s]on ventre» (82).

Des poèmes en prose et en italique, plus réflexifs et introspectifs, ponctuent le recueil et en donnent la mesure. Loin d'être un signe de pauvreté, le recours à la simplicité y apparaît comme un gage de justesse: «Je ne savais pas que les objets, quand on s'y attarde de façon sensible, pouvaient rendre la matière véritable d'une émotion dont j'essaie de dévoiler les plis», lit-on à la page 22. Le moins qu'on puisse dire est que cette découverte est mise à profit:

Si vous me demandez où je suis maintenant,

c'est moi qui essaie d'écrire de quoi de beau avec

le mouillé de la zamboni. (23)

Chaque pensée est un crash

de corneilles dans un blender

une matière nouvelle à dompter. (61)

On pourrait multiplier à l'envi les exemples de poèmes de trois vers saisissants d'éloquence, propres à rendre envieux le plus habile des haikistes, tant l'auteure excelle à faire dialoguer la poésie des objets et celle de la langue. Et c'est ainsi qu'elle parle de poésie, en ayant l'air de ne pas y toucher, alors qu'il s'agit pour elle d'une question de survie $^{3}$. On ne s'étonne donc pas que ce qu'il reste de plus significatif de cet amour manqué, de ce si beau ratage, soient des souvenirs modestes: un lit de sapinage, un instant heureux à la pêche, des vêtements dans une chambre, une odeur de peau.

S'amusant à se déplacer, comme si elle jouait à cache-cache avec le lecteur', c'est finalement au bord du fjord qu'elle choisit de disparaître. Là, dans un endroit secret, elle plante sa tente, se laisse dissoudre dans le paysage pour mieux se ressaisir, se rassembler. Si elle ne perd pas contact avec sa blessure, l'expérience qu'elle fait alors du paysage est salutaire, dont le dernier poème en prose fait le récit:

Je me touche, je lis, un écureuil essaie de me grimper dessus - je suis une princesse Disney en tabarnak. Je marche dans la forêt dense, je m'égratigne partout et

2 «Je suis hanté par tout l'espace que je vais vivre sans toi:/ce câlique de vers de Brautigan.» (36; l'auteure souligne.)

3 «Toujours en train d'écrire de quoi pour survivre,/j'invente des listes de choses à faire, déconstruis/les structures fanées de rêves dociles: oignons/revenus et soupes chaudes, chanterelles et tartes/aux pommes; nos accidents de bonheur simple.» (17)

4 Comme l'indique la formule "si vous me demandez où je suis", qui revient à quelques reprises, dont la dernière déclinaison éclaire le titre du recueil: «si vous me cherchez, je suis chez nous, /ou quelque part sur Nitassinan,/toutes mes portes et mes fenêtres sont ouvertes//je chauffe le dehors» (84). 
j'aime ça. Vraiment, j'aime que mon corps se magane par le hors-piste, qu'il ait des traces comme des signes de fierté et d'autonomie, de force et d'endurance. Dans ces moments-là, je suis toute là, pas tuable - pas grand-chose et totale à la fois. [...] Le dehors est la seule réponse que j'ai trouvée au-dedans. (65-66; les italiques sont de l'auteure.)

Cet extrait ouvre sur une suite où les poèmes sont plus apaisés. La narratrice y apparaît pleinement en phase avec le vivant, forte de sa «chaleur de femme» (72) qui tape des sentiers où l'absent à son tour disparaît, absorbé par la neige. Elle avance tête haute, épaules dégagées, laisse «le territoire [l]'éparpiller comme les oiseaux/migrateurs savent pas se perdre» (80), désillusionnée mais nullement désespérée, "[a]vec pour allié le mot lentement» (76).

Laconiques mais ô combien efficaces, les poèmes de Marie-André Gill prêtent davantage à la méditation qu'aux commentaires. Ils vous laissent au silence, plein de gratitude, et continuent de vibrer en vous, tel un paysage sauvage bouleversant de beauté, dont vous seriez le seul témoin, mais qui vous donne cette grisante impression de communier avec l'univers. De ces paysages où l'on se dit qu'on pourrait soimême volontiers disparaître.

Dans Misère et dialogue des bêtes ${ }^{5}$, Jean-Marc Desgent poursuit dans la voie qu'il a empruntée depuis Vingtièmes siècles ${ }^{6}$, livre marquant qu'il n'avait, à mon avis, pas encore égalé. Si l'on reconnaît la manière et le ton auxquels il nous a habitués depuis, quelque chose de singulier se produit dans ce livre qui le rend digne de mention: on y assiste à une véritable rencontre, en l'occurrence celle d'un loup.

J'avance à grands pas je souris en marchant une tête m'est arrivée m’arrive un loup qui attend la fin du monde il peut s'il le veut m'avaler il désire un regard il meurt vieux comme moi étranger à chaque arbre. (12)

Notons la formulation: un loup m'arrive, comme on le dirait d'un événement. Cette rencontre est en effet un événement, plus encore, un avènement pour le sujet qui en sera complètement transformé.

Comme dans Chauffer le dehors mais inversement, des poèmes en prose sont ponctués par des poèmes en vers et en italique plus réflexifs et introspectifs. D'entrée de jeu, la clé de lecture est donnée : l'ensauvagement de la langue, de toutes les langues. Parallèlement, un mouvement de chute est accusé qui persiste jusqu'à la fin du recueil: "Fou tombé mauvais» (8); "nous sommes un petit corps noirci tombé malade en lui-même» (9), "les ruisseaux vont à leur point de chute» (15; les italiques sont de l'auteur), etc. On entre ici en poésie comme on entre en forêt, où langage et animalité s'assaillent, combattent, s'enroulent l'un sur l'autre (on pense à

5 Jean-Marc Desgent, Misère et dialogue des bêtes, Montréal, Poètes de brousse, 2019, 47 p.

6 Jean-Marc Desgent, Vingtièmes siècles, Trois-Rivières, Écrits des Forges, 2005, 64 p. 
l'hélice d'ADN), comme jetés au «cirque des rituels» (13). À mesure qu'il s'enfonce dans la forêt, pris à ce tourbillon, le corps du sujet se dissipe et se mêle à la matière qui s'échauffe et se liquéfie. Ça mouille, ça régresse, jusqu'à la glaise, jusqu'à l'eau originelle où l'on se noie:

les branches me giflent me griffent le dos me mordent le cou à ciel ouvert mon orage n'a plus le sens que je voulais je le transforme en eaux intérieures en plaintes terrestres mon front touche l'herbe et les roches trempées je me suis donné un défi qui ne trahit pas je suis entré dans la maison cassée j'ai la pensée colonne une animalité verticale (14).

Tandis que chez Gill la forêt est bienveillante, la hiérarchie absente, ici la forêt se fait d'abord hostile, et la hiérarchie est à déconstruire. Le poète doit se soumettre à la catastrophe, au grand sacrifice s'il veut renaître à cette rencontre qui décuple puis perd son identité avant de le plonger dans la profondeur du temps, remuant cadavres et fantômes:

Certains cadavres sont questionnés entendus compris d'autres sont muets malgré les autopsies répétées ce sont nos êtres ceux debout ceux couchés déformés par l'arthrose crâne de la faim crâne de la soif je ne resterai pas certitude du cœur arrêté je suis déjà un mal accroché je feuillette mon livre de passage ce sont des pages la même catastrophe tombée de nous. (16)

Le sens se déploie par déflagration, par contamination. Telle une traînée de poudre, il va en tous sens, grouille, exubère, prolifère. Impossible à contenir, la langue ne va jamais sans reste. Or c'est précisément ce reste que recueille le poème, c'est de ce reste, ce mal défini, mal dégrossi du sens qu'il tire sa matière, comme c'est de cadavres que l'animal se nourrit ${ }^{7}$. Chaque chose vient avec son contraire, comme entraînée vers sa disparition; le temps est cacophonique, toutes époques confondues, comme autant de dimensions, de versions du réel se chevauchant.

En prenant part à cette nature déchaînée où l'animalité l'altère, le sujet perd jusqu'au fil des lignées maternelle, paternelle, dans lesquelles il ne se reconnaît plus. Ce n'est pas tout de perdre son humanité, encore faut-il «réussir sa propre disparition» (20):

ça fait des années que je suis une faïence une brisure annoncée un lit saccagé par les labours inutiles je répète je dis cent fois les mêmes mots je me refuse le souffle le soleil un certain vertige létal je construis igné pièce par pièce miniature par miniature des enfants brouillons (21);

L'animal un brouillard passe et inversement m'est apparu dans les feux que j'ai allumés dans les trous d'enfantement. (23)

7 «Quand je me suis approché de l'âge des bêtes quelque chose a déchargé sa carabine en moi.» (46) 
Ce brouillard est aussi un non-temps où le sujet devient à la fois brouillon d'enfant et «momie de l'homme» (22). La langue elle-même ne rend plus que des bruits "quasi inhumains» (25), matière sonore qu'on ne peut plus saisir qu'avec ses mains. Mais c'est par le toucher (des pieds, des mains) qu'une fois rendu à son terme, le processus d'ensauvagement pourra s'inverser:

comme si la fatigue de marcher
nous rendait étranges étourdis
je finirai par trouver un endroit
où m'asseoir où je pourrai toucher
à moi en me reconnaissant (25; les italiques sont de l'auteur);

je passe entre deux paysages je ressemble aux nuages ça se déplace bicéphale ça bouge féminin ça bouge masculin ici autrefois la vie la langue était marchée de bout en bout (33).

Au point de croisement ou de revirement, qui est le point de rencontre, le loup apparaît moins comme un objet de fascination que comme une bête sauvage. Or c'est en s'éprouvant viande crue que le sujet se reconnaît humain. En lui révélant sa vulnérabilité, le loup lui redonne son identité:

Une fois traversé passé le grand trou on appartient à la beauté générale je me suis habillé noir je me suis redevenu et j'ai embrassé tout ça j'ai fait miracle avec le tremblement le plaisir de mordiller la peur de détruire les horreurs de la table dressée en plein bois voilà pourquoi l'être se tient plusieurs il entre à l'origine et à l'événement.

(44; je souligne.)

$\bar{A}$ la fois point de convergence de la mort et de la naissance et lieu de tous les combats, ce grand trou noir de l'origine ne pourrait-il pas aussi bien être la gueule du loup? Entre l'homme et le loup, qui est le bourreau, qui, la victime? Rien dans ce livre ne permet de trancher: "tuer être tué ce n'est pas exaltant c'est pourtant ce qui demeure» (45). Tel un Jonas postmoderne, c'est peut-être en passant par le ventre du loup - en prenant part à l'événement de sa dévoration - que le sujet peut remonter jusqu'à la nuit des temps et retourner aux fondements de son humanité.

Incidemment, c'est sur un désir de «[r]evenir», de "réapprendre à marcher» en forêt sans danger que se termine le livre, désir de «reprendre en clichés noir et blanc [en mots?]/ce qu'on a laissé là-bas » (48), mais en reconnaissant que tout cela, le loup, le lynx et consorts, «la terre et son contraire/le bleu et son évidence/ne me sont pas étrangers» (48). Si accepter son animalité - sans en faire un objet de répulsion ou de fascination - , c'est lui laisser frayer son chemin en nous, réussir sa disparition, ce serait peut-être se laisser traverser par la langue redevenue matière, comme une forêt amie. 
Depuis Des ombres portées ${ }^{8}$, Paul Chanel Malenfant a fait de la mort un thème de prédilection ${ }^{9}$. Si la vie peut en quelque sorte vous imposer le sujet - il a écrit successivement sur le décès d'une amie, du père, de la mère -, il n'est pas donné à tous les écrivains de tresser avec un tel doigté la hideur et la beauté de la mort. Il n'y a plus d'après est un livre bouleversant, et certainement l'un des meilleurs de l'auteur. Malenfant est un as de la délicatesse et du raffinement. Sans faire de compromis sur le travail de la forme, il approche ici la mort avec une certaine impudeur et va très loin dans l'expression de la douleur. Une constante tension entre déchirement et apaisement, révolte et gratitude traduit avec beaucoup de justesse, jusque dans les moindres replis de la langue, le vertige que l'on ressent quand la mort vous tient dans son étau et ne vous lâche plus.

Le livre fait le récit de la perte de l'ami, amant, amour de toute une vie. Comme chez Gill, le poème emprunte la forme de l'adresse, invitant au rapprochement tout en exacerbant le sentiment d'éloignement, ce qui fait résonner le silence de l'absent. L'adresse est un appel, une prière, à entendre ici au sens littéral autant que métaphorique - les références religieuses étant nombreuses. En vertu de l'intimité qui les lie, la mort de l'ami acquiert des propriétés spéculaires, lui rappelant sans cesse que «tous les hommes sont condamnés» (14): «J'assiste à travers toi/Ā ma propre disparition.» (29) Alors que la mort du père et de la mère mettait à mort l'enfant, ici $c^{\prime}$ est l'homme - l'ami, l'amant, l'amoureux - qui apprend à disparaître ${ }^{10}$ :

Je ne savais rien de la mort

Avant la tienne (93);

en âge d'homme je suis

souffrance monacale

orphelin de tous (138).

La maladie affectant de près l'expression du mourant ${ }^{11}$, elle agit également sur la langue du poète, qui dès lors ne s'y reconnaît plus:

La syntaxe est broyée (17);

Je nous parle aux sons (19);

je ne sais plus qui je suis, je ne sais plus

de quelle parole je te parle (20);

Nous partageons l'écume de la langue. (37)

8 Paul Chanel Malenfant, Des ombres portées, Montréal, Éditions du Noroît, 2000, 140 p.

9 Il y revient d'ailleurs dans son dernier recueil: «J'ai écrit tant de livres/tombeaux/sur vos ombres portées/ depuis la mort de Lise/l'amoureuse amie.» Paul Chanel Malenfant, Il n'y a plus d'après, Montréal, Éditions du Noroît, 2019, p. 69.

10 D'ailleurs il parle de la mort au «nous»: «Saura-t-elle un jour, Edna Stern la pianiste, comment allemande courante et sarabande, elle nous a aidés à mourir?» (27; les italiques sont de l'auteur)

11 «le verdict est tombé:/cancer de la langue et de la gorge» (43). 
L'écume de la langue, serait-ce ce que recueille le poème lorsque, devant les nécessités de la communication, les mots sont dépouillés de leur pouvoir symbolique et ramenés aux origines du sens ${ }^{12}$, processus au terme duquel une autre force d'évocation se fait jour? C'est ainsi que les noms de médicaments, mis bout à bout, se muent en poème exploréen.

L'expérience de la mort a comme propriété de dilater le temps («le temps n'est plus du temps» [21]). Le poème donne à ressentir l'extrême lenteur de l'agonie, exactement comme dans Les heures de Fernand Ouellette ${ }^{13}$, auquel le livre fait écho à plusieurs égards et dont on peut supposer qu'il en a accompagné l'écriture ${ }^{14}$. Comme chez Ouellette, on remarque une véritable transitivité de la mort, malgré son caractère insaisissable. Rien n'est caché du corps agonisant, de sa débilité, et cependant il n'y a là ni sensationnalisme ni complaisance dans la trivialité. On assiste au labeur de la mort... et on s'y reconnaît. Comme le loup de Desgent, la mort nous arrive.

La trajectoire de l'amoureux (il était né en 1930) a couvert une bonne partie du $x^{e}$ siècle. Aussi la petite histoire de cette figure tutélaire - de celles à qui l'on pense "comme à un prophète» (32) - se dessine sur fond de la grande Histoire dont elle a été témoin. La préparation aux adieux est l'occasion d'une rétrospective, sorte de bilan du siècle. On traverse ainsi l'histoire du Québec et celle de la France, de la maison familiale de Rimouski jusqu'aux paysages de Sainte-Luce-sur-Mer, en passant par Saint-Germain-des-Prés, où se retrouve «un jeune homme en fleur/dans la peau d'un homme/dans la force de l'âge» (84). Les souvenirs s'enchaînent qui ont vu naître cette intimité clandestine (il y a un très bel et touchant érotisme dans ces poèmes), puis s'épanouir ce grand amour. Les espaces, les événements et les temps se mélangent, les articulations procédant par associations, comme en rêve. Les motsimages s'appellent, se bousculent, se télescopent; à mesure que les sens échouent, le réel perd de son épaisseur ou apparaît sous un jour différent. Ainsi l'hôpital se superpose aux camps nazis, formant une sorte de palimpseste de la déréliction. La guerre devient une métaphore du combat à mener pour traverser la mort et entrer - peut-être? - dans l'éternité:

Je suis un vieux soldat de plomb

sur l'échiquier de l'enfance;

je veille au froid des sous-bois

parmi les ronces. (39)

Comme chez Desgent, ce maelström spatio-temporel mène à un trou noir où il n'est plus possible de distinguer la victime du bourreau:

12 Notons que les appels aux mères sont nombreux dans le livre.

13 Fernand Ouellette, Les heures, Montréal/Seyssel, Éditions de l'Hexagone/Champ Vallon, 1987, 118 p.

14 Rappelons que Paul Chanel Malenfant est un spécialiste et fervent lecteur de Fernand Ouellette, à qui il avait consacré une partie de sa thèse de doctorat. Voir Paul Chanel Malenfant, La partie et le tout. Lecture de Fernand Ouellette et de Roland Giguère, Québec, Presses de l'Universitê Laval, coll. «Vie des lettres québécoises", 1983, 397 p. 
Tu ne seras plus qu'un souffle sans voix

Une voce poco fa? parmi les galaxies

dérivant aux trous noirs de l'origine

du monde. Nous ignorons

qui est la victime, qui est le bourreau,

qui de Dieu ou de Diable

nous a conduits aux terrains vagues (42).

Le mourant, ayant une foi que son amoureux, de vingt ans son cadet, ne partage pas («tu étais de la foi/de Thomas d'Aquin/j'étais du pari/de Blaise Pascal» [80]), est présenté comme une figure christique. D'où cette colère contre Dieu. Mais si l'on y songe, n'est-ce pas ce qui nous est le plus étranger chez les êtres qu'on aime qu'il nous reste de plus précieux lorsqu'ils nous ont quittés, et qui continue à faire énigme, comme une question qu'ils nous adresseraient par-delà la mort? Aussi suis-je portée à entendre cet appel à la révolte comme une prière - fût-elle maudite ${ }^{15}$ - ou à tout le moins comme un pari. D'ailleurs c'est le Dieu de Job ${ }^{16}$, celui de l'Ancien Testament, qui se dresse ici en opposant - un dieu vengeur, qui nous tient en otages et nous mène à l'abattoir. Le peuple des «amoureux des hommes» (84), condamné à la clandestinité en dehors des grandes villes (le Bas-du-Fleuve est désigné comme "terre de Caïn"), est identifié au peuple juif, doublement sacrifié, exilé et errant en quête de sa Jérusalem.

Cette mort est une rafle: elle fauche tout, exacerbe le manque, fait retentir le «silence/monochrome/de Dieu» (117). Emportant jusqu'aux souvenirs («que ferai-je/de nos photographies?» [126; les italiques sont de l'auteur]), elle laisse le survivant «dans l'émerveillement étonné/désespéré d'être vivant» (70). Il lui reste le fleuve, toujours pareil, toujours différent, les lieux familiers devenus étrangers, la «maigre consolation des choses» (140) et les livres - ceux qui accompagnent le récit ${ }^{17}$ et les autres. Sur ce fond de paysage marin, un passage se détache qui rappelle les livres de Gill et de Desgent:

\author{
Nous avançons \\ parmi les arbres \\ seuls au monde \\ moderne \\ entre les capitaux \\ les affaires étrangères \\ nous marchons \\ dans le secret \\ des sous-bois. (123)
}

15 «Écoute ô mon amour grabataire/ma prière maudite/silencieuse telle/la musique atonale de la terre.» (77)

16 «En marge de l'opprobre,/de la déréliction,/Job somnole en silence/sur son tas d'immondices.» (57)

17 Comme il lui est fréquent de le faire, l'auteur multiplie les références et les citations, dont la plupart des sources sont données à la fin de l'ouvrage. 
Dans leur infinie lenteur, leur modeste bienveillance, les arbres seraient-ils nos plus précieux alliés, et les sous-bois des lieux propices aux amours naissantes, qu'ils protègent de «la violence du monde» (35)? Géants fragiles, incarnant à la fois la naissance, la mort et l'espace d'une vie qui les sépare et les relie, les arbres sont en quelque sorte des gardiens de l'origine; nous y rappelant, ils nous rappellent du même coup à notre devoir d'humilité.

À l'occasion d'un entretien qu'il accordait récemment à Georges Leroux, Paul Chamberland parlait de la poésie comme moyen de résistance ${ }^{18}$. En réponse à Leroux, qui s'étonnait de le voir retourner aux fonctions du langage de Roman Jakobson, Chamberland a invoqué la nécessité de revenir à la fonction anthropologique de la poésie, laquelle nous garderait de l'inhumain. Il me semble de plus en plus évident que chez les écrivains dont les œuvres sont fortes, la poésie exerce ce pouvoir de résistance et même s'impose comme moyen de survie, et en cela ils actualisent cette fonction anthropologique dont parle Chamberland. Dans les livres recensés ici, cela s'exprime en partie par un processus d'ensauvagement impliquant un accueil des diverses formes de vie qui nous composent et dans lequel le paysage joue un rôle de premier plan. Cela me rappelle une très intéressante réflexion de Giorgio Agamben sur l'être "en état de paysage», que je citerai en guise de conclusion, car il y décrit bien, il me semble, ce qui se joue dans la poésie actuelle lorsqu'elle se laisse affecter par l'éventualité de notre disparition:

L'être, en état de paysage, est suspendu et désactivé, et le monde, devenu parfaitement inappropriable, va pour ainsi dire au-delà de l'être et du néant. Ni animal ni humain, celui qui contemple le paysage est seulement paysage. Il ne cherche plus à comprendre, il se contente de regarder. Si le monde était le désœuvrement du milieu animal, le paysage est, pour ainsi dire, désœuvrement du désœuvrement, être désactivé. Ni désinhibiteurs animaux ni étants, les éléments qui forment le paysage sont ontologiquement neutres. Et la négativité qui, sous la forme du néant et de la non-ouverture, était inhérente au monde - puisque celui-ci provient de la fermeture animale dont il n'était que la mise en suspens - est désormais congédiée ${ }^{19}$.

18 Entretien public présenté à l'occasion de la sortie de son essai Le dire vrai du poème (Montréal, Éditions du Noroît, coll. «Chemins de traverse», 170 p.), Librairie Gallimard, Montréal, 8 mai 2019.

19 Giorgio Agamben, Homo Sacer, vol. 4.2: L'usage des corps, traduit de l'italien par Joël Gayraud, Paris, Éditions du Seuil, coll. «Ordre philosophique», 2015, p. 141. 